\title{
Artificial Neural Networks to Predict the Power Output of a PV Panel
}

\author{
Valerio Lo Brano, Giuseppina Ciulla, and Mariavittoria Di Falco \\ DEIM Università degli studi di Palermo, Viale Delle Scienze, Edificio 9, 90128 Palermo, Italy \\ Correspondence should be addressed to Valerio Lo Brano; lobrano@dream.unipa.it
}

Received 28 May 2013; Accepted 29 November 2013; Published 23 January 2014

Academic Editor: David Worrall

Copyright (C) 2014 Valerio Lo Brano et al. This is an open access article distributed under the Creative Commons Attribution License, which permits unrestricted use, distribution, and reproduction in any medium, provided the original work is properly cited.

\begin{abstract}
The paper illustrates an adaptive approach based on different topologies of artificial neural networks (ANNs) for the power energy output forecasting of photovoltaic (PV) modules. The analysis of the PV module's power output needed detailed local climate data, which was collected by a dedicated weather monitoring system. The Department of Energy, Information Engineering, and Mathematical Models of the University of Palermo (Italy) has built up a weather monitoring system that worked together with a data acquisition system. The power output forecast is obtained using three different types of ANNs: a one hidden layer Multilayer perceptron (MLP), a recursive neural network (RNN), and a gamma memory (GM) trained with the back propagation. In order to investigate the influence of climate variability on the electricity production, the ANNs were trained using weather data (air temperature, solar irradiance, and wind speed) along with historical power output data available for the two test modules. The model validation was performed by comparing model predictions with power output data that were not used for the network's training. The results obtained bear out the suitability of the adopted methodology for the short-term power output forecasting problem and identified the best topology.
\end{abstract}

\section{Introduction}

Among renewable energy sources (RES), solar energy has the greatest energy potential and PV arrays permit to produce electric power directly from sunlight; furthermore, during the operational phase, the energy production occurs without fossil-fuel consumption or noise, and not posing health and environmental hazards. These features will make the PV devices one of the most important among the technologies based on the exploitation of RES [1-5]. Nevertheless, the technological and environmental benefits of PV technology are hindered by economic and technical factors. The high cost of production and installation make the PV technology feasible to the customer only if there are public funding opportunities. Furthermore, there are various concerns associated with PV modules, such as the impact of their interconnection to the grid [6]. Some studies have been carried out on this, for example, [7], but, in general, there is little information on the topic. The most severe disturbance caused by the connection of a large amount of PV generation to the grid would be encountered when a band of cloud sweeps over an area with a large concentration of PV generators. This could result in a fairly large and sudden variation in the PV output. The condition would be aggravated if this change in irradiance occurred during a rapid increase in load [8]. For these reasons, it is clear that the availability of reliable predictive tools is very important for the dissemination of $\mathrm{PV}$ technologies, to optimize the performance of PV systems in the planning and operational phase and finally to correctly assess the economic return. In order to evaluate the real performance of PV panels is very important the correct prediction of power output; an increase of even a few degrees of the PV panel together with a lower solar irradiance can considerably reduce the conversion efficiency of the system thus reducing the power output [9]. Indeed, an important consideration in achieving the efficiency of a PV panel is to evaluate the performance for any weather conditions and to match the maximum power point. Many methods based on the MPPT (maximum power point technique) have been reported in the literature, many others applied empirical 


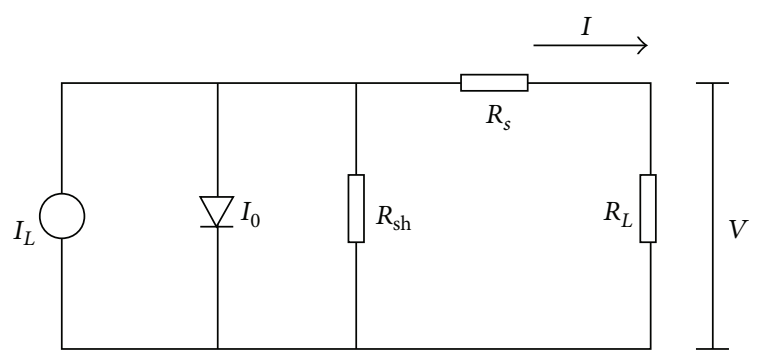

FIGURE 1: One diode simplified equivalent circuit for a solar cell closed on a resistive load $R_{L}$.

correlations to evaluate the thermoelectrical performance of a PV system. However, these approaches require detailed knowledge of physical parameters of the PV system and manufacturing specifications. Another approach is represented by adaptive systems. An adaptive system is a system that is able to adapt its behaviour according to changes in its environment or in parts of the system itself. An adaptive system, such as artificial neural networks (ANN), does not require any physical definitions for a PV array but should allow predicting, in a fast and reliable procedure, the power output of the PV module varying the weather conditions. This paper presents a comparison of different types of ANNs that better forecasts the PV power output. The authors have tested the use of ANN to predict the power output of a PV panel using the data monitored in a test facility.

\section{The Power Output of a PV Module}

To design and assess the performances of a PV system, an accurate PV model should predict a reliable current-voltage $(I-V)$ and power-voltage $(P-V)$ curves under real operating conditions.

The "five-parameters model" represents the most common equivalent circuit that better describes the electrical behaviour of a PV system. The equivalent circuit is composed of a photocurrent source $I_{L}$, a diode in parallel with a shunt resistance $R_{\mathrm{sh}}$, and a series resistance $R_{s}$ as shown in Figure 1 .

Based on this simplified circuit, the mathematical model of a photovoltaic cell can be defined in accordance with the following expression that permits to retrieve the $I-V$ curve:

$$
I=I_{L}-I_{0}\left(e^{\left(V+I \cdot R_{s}\right) / n T_{c}}-1\right)-\frac{V+I \cdot R_{s}}{R_{\mathrm{sh}}},
$$

in which $I_{L}$ depends on the solar irradiance, $I_{0}$ is the diode reverse saturation current and is affected by the silicon temperature, $n$ is the ideality factor, and $T_{c}$ is the cell absolute temperature.

As it is known, the performance of a photovoltaic panel is defined according to the "peak power," which identifies the maximum electric power supplied by the panel when it receives a solar irradiance $G$ of $1 \mathrm{~kW} / \mathrm{m}^{2}$ at a cell temperature of $25^{\circ} \mathrm{C}$. For given values of $G, T_{c}$ and $R_{L}$, the operating point can be identified by drawing lines of the different loads $R_{L}$ on the $I-V$ characteristic (Figure 2); the maximum power points are indicated by red circles.



(a)

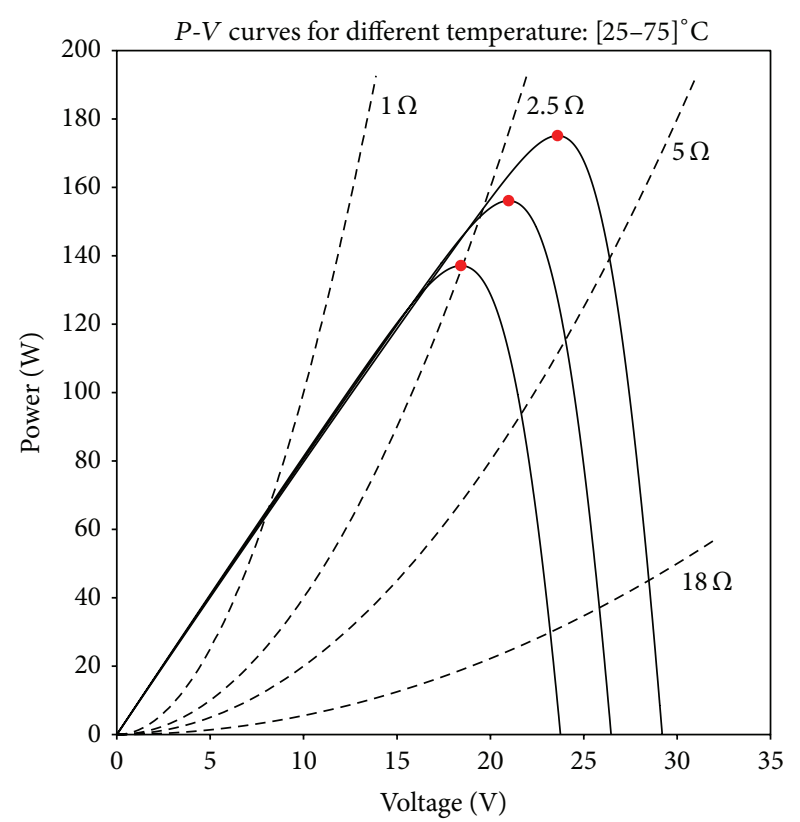

(b)

FIGURE 2: Working point of a generic PV panel at constant temperature $\left(25^{\circ} \mathrm{C}\right)$, varying solar irradiance $\left(1000-200 \mathrm{~W} / \mathrm{m}^{2}\right)$, and electric load (a) and at constant irradiance $\left(1000 \mathrm{~W} / \mathrm{m}^{2}\right)$, varying temperature $\left(25-75^{\circ} \mathrm{C}\right)$, and electric load (b).

In actual conditions, it is essential to evaluate the operating condition under all possible circumstances of $G, T_{c}$, wind speed $W$, air temperature $T_{\text {air }}$, and electric load $R_{L}$. The $T_{c}$ temperature thus is a key parameter that affects the energy conversion efficiency of a PV panel: increasing the temperature decreases the delivered power.

Furthermore, in the literature, it is possible to find different algorithms for seeking the maximum power point 
[10-12]. In detail, the indirect methods have the particular feature that the MPP is estimated from the measures of the $\mathrm{PV}$ generator's voltage and current $\mathrm{PV}$, the irradiance, or using empiric data, by mathematical expressions of numerical approximations. In the most of the maximum power point tracking (MPPT) methods, described in the literature, the optimal operation point of a generic PV system is estimated by linear approximations $[13,14]$ as

$$
V_{\mathrm{mpp}}=k_{v} \cdot V_{\mathrm{OC}} \text { or } I_{\mathrm{mpp}}=k_{i} \cdot I_{\mathrm{sc}},
$$

where $V_{\mathrm{mpp}}$ and $I_{\mathrm{mpp}}$ are the maximum voltage and current, respectively, $k_{v}$ and $k_{i}$ are two constants of proportionality (voltage and current factors) dependents on the characteristics of the PV array used, $V_{o c}$ is the open circuit voltage, and $I_{\mathrm{sc}}$ is the short circuit current.

Nevertheless, the direct methods can also be used; they offer the advantage that they obtain the actual maximum power from the measures of the PV generator's voltage and current PV. In that case, they are suitable for any irradiance and temperature [15]. All algorithms, direct and indirect, can be included in some of the DC/DC converters, maximum power point tracking (MPPTs), for the stand-alone systems [10].

Recently, the fuzzy logic controllers (FLCs) and artificial neural network (ANN) methods have received attention and increased their use very successfully in the implementation for MPP searching [16-26]. The fuzzy controllers improve control robustness and have advantages over conventional ones. They can be summarized in the following way [27]: they do not need exact mathematical models, they can work with vague inputs and, in addition, can handle nonlinearities, and are adaptive, in nature; likewise, their control gives them robust performance, under parameter variation, load and supply voltage disturbances. Based on their heuristic nature and fuzzy rule tables, these methods use different parameters to predict the maximum power output: the output circuit voltage and short circuit current [17]; the instantaneous array voltage and current [18-20]; instantaneous array voltage and reference voltage (obtained by an offline trained neural network) [16]; instantaneous array voltage and current of the array and short circuit current and open circuit voltage of a monitoring cell $[21,22]$ and solar irradiance, ambient temperature, wind velocity and instantaneous array voltage and current, used in $[23,25,26]$.

Next, three different ANNs are proposed with the aim to forecast power output of PV modules.

\section{Generalities on Adaptive and ANN Systems}

Adaptive systems and ANNs are nonlinear elaboration information systems whose operation function draws its inspiration by biological nervous system. When there is no clear relationship between the inputs and outputs, it is not easy to formulate the mathematical model for such as system; on the contrary, the ANN can model this system using samples [27].

Their ability to learn from experimental data makes ANN very flexible and powerful than any other parametric approaches. Therefore, neural networks have become very

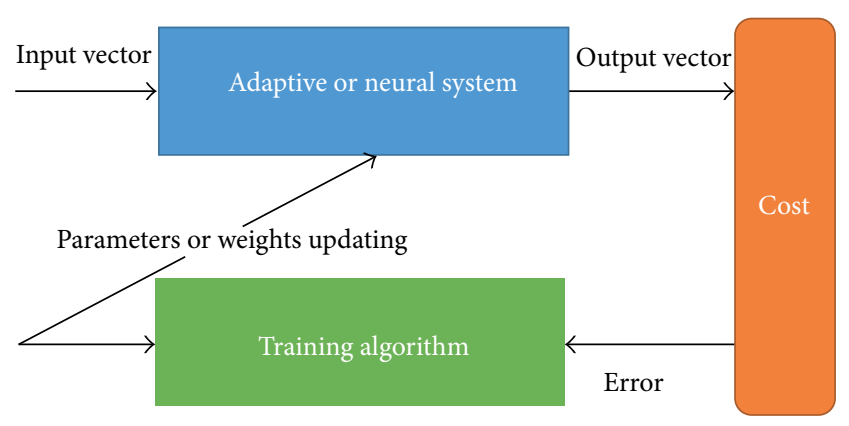

Figure 3: Adaptive or neural system's design.

popular for solving regression and classification problems in many fields [28]. Because the neural network does not require any detailed information about the system or process, it operates like a black box [29].

\section{The Artificial Neuron}

An ANN consists of many interconnected processing nodes known as neurons that act as microprocessors (Figure 3).

Each artificial neuron (Figure 4) receives a weighted set of inputs and produces an output.

The activation potential $A_{i}$ of an $\mathrm{AN}$ is equal to

$$
A_{i}=\sum_{j=1}^{N} w_{i j} x_{j}-b_{j},
$$

where $N$ is the number of elements in the input vector $x_{i}$, $\omega_{i j}$ are the interconnection weights, and $b_{i}$ is the "bias" for the neuron [30]; the bias is a coefficient that controls the activation of the signal handled by the AN. The neuron output depends only on information that is locally available at the neuron, either stored internally or arrived via the weighted coefficients.

\section{The Activation Function}

The neuron output $y_{i}$ is calculated by the summation of weighted inputs with a bias through an "activate on function" as follows:

$$
y_{i}=\Phi\left(A_{i}\right)=\Phi\left[\sum_{i=1}^{N} \omega_{i j} x_{i}-b_{i}\right] .
$$

The activation function is intended to limit the output of the neuron, usually between the values $[0,1]$ or $[-1,+1]$. Typically it is used the same activation function for all neurons in the network, even if it is not necessary [31]. The most commons activate functions are the step function, the linear combination, and the sigmoid function as shown in Figure 5.

In the step function, the output $\Phi\left(A_{i}\right)$ of this transfer function is binary, depending on whether the input meets 


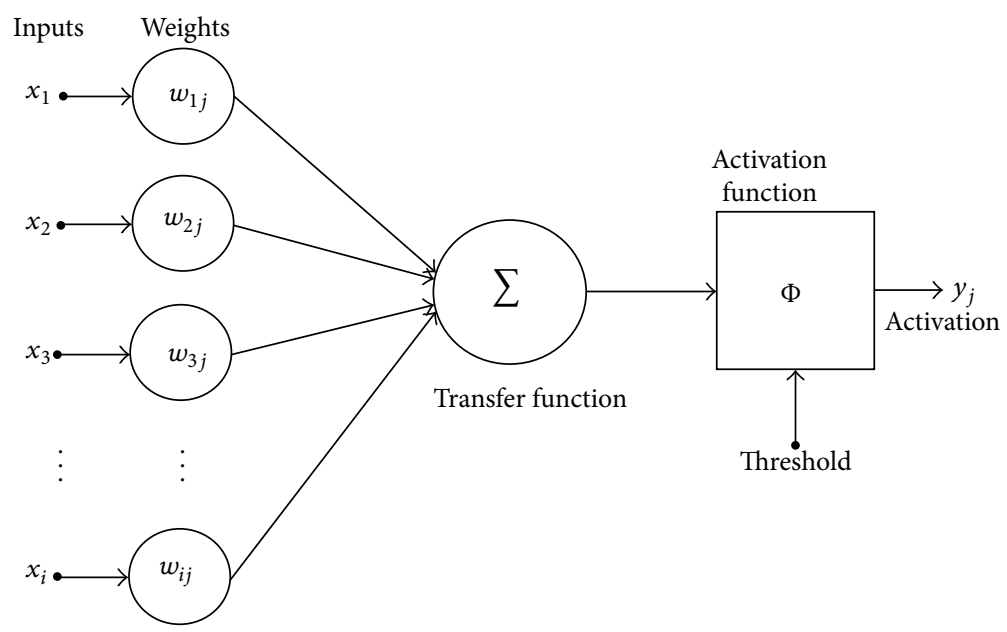

FIGURE 4: Schema of artificial neuron.



(a)



(b)



(c)

FIgURE 5: The most common activation functions: (a) step function; (b) linear function; (c) sigmoid function.

a specified threshold. The "signal" is sent; that is, the output is set to one, if the activation meets the threshold:

$$
y_{i}=\Phi\left(A_{i}\right)= \begin{cases}1 & \text { if } A \geq \text { threshold } \\ 0 & \text { if } A<\text { threshold. }\end{cases}
$$

The step activation function is especially useful in the last layer of an ANN to perform a binary classification of the inputs.
A linear combination, usually more useful in the first layers of an ANN, where the weighted sum input of the neuron plus a linearly dependent bias becomes the system output. A number of such linear neurons perform a linear transformation of the input vector as

$$
y_{i}=\Phi\left(A_{i}\right)=k A_{i},
$$

in which $k$ is a scale parameter. 
A sigmoid activation function produces an output value between 0 and 1 . Furthermore, the sigmoid function is continuous and differentiable. Due to these reasons, this activation function is used in ANN models in which the learning algorithm requires derivatives. Often, sigmoid function refers to the special case of the logistic function defined by the formula

$$
\Phi\left(A_{i}\right)=\frac{1}{1+e^{-k A}},
$$

where $k$ is a constant that control the shape of the curve. The sigmoid function such as the logistic function also has an easily calculated derivative, which can be important when calculating the weight updates in the network. It thus makes the network more easily mathematically manipulable and was attractive to early computer scientists who needed to minimize the computational load of their simulations.

\section{Architecture or Topology of an ANN}

Generally, an ANN is usually divided into three parts: the input layer that collects the inputs $x_{i}$, the hidden layer $h_{i}$, and the output layer that issues the outputs $y_{i}$. If a neural network is composed by a single layer of unidirectional connections from the input nodes to output nodes is called Perceptron.

This configuration is the simplest and is not able to solve not linearly separable problems. For these kind of complex problems is more useful to use a multilayer perceptron (MLP) ANN that is a feed forward ANN model that maps sets of input data onto a set of appropriate outputs. The feed forward was the first and arguably simplest type of ANN developed. In a feed forward ANN the connections between the units do not form a directed cycle; the information moves in only one direction, forward, from the input nodes, through the hidden nodes (if any) and to the output nodes. By this way, there are no cycles or loops in the network.

According to the above definitions, a feed forward MLP consists of multiple layers of nodes in a directed graph, with each layer fully connected to the next one. Except for the input nodes, each node is a neuron (or processing element) with a nonlinear activation function.

On the contrary, a radial neural network (RNN) is a class of neural network where connections between units form a directed cycle. This creates an internal state of the network that allows the ANN to exhibit a dynamic behaviour. Unlike feed forward ANN, RNNs can use their internal memory to process arbitrary sequences of inputs. This makes them applicable to tasks such as the recognition of time series, where they have achieved the best known results.

\section{Training Algorithm}

Before the neural network can be used to a specific problem, its weights have to be tuned. This task is accomplished by the learning process in which the network is trained. This algorithm iteratively modifies the weights until a specific condition is verified. In most applications, the learning algorithm stops when the error between desired output and the calculated output produced by the ANN reach a predefined value. The error is updated by optimizing the weights and biases. After the training process, the ANN can be used to predict the output parameters as a function of the input parameters that have not been presented before. An epoch is a collection of all available samples; it is also the term used for a training iteration of the system: when one epoch has passed, the adaptive system has been presented with the available data once. As adaptive systems are for the most part trained iteratively, many epochs are usually required to fully train a system.

Concerning the learning algorithm, there are generally two typologies of ANN learning algorithm [32]:

(i) supervised learning;

(ii) unsupervised learning.

Supervised learning is characterised by a training set composed of pairs of inputs and corresponding desired outputs. The error produced by the ANN is then used to update the weights (back propagation).

In unsupervised learning algorithms, the network is only provided with a set of inputs and without desired output. The algorithm guides the ANN to self-organize and to adapt its weights. This kind of learning is used for tasks such as data mining and clustering, where some regularities in a large amount of data have to be found.

The information in the previous layers obtained by updating the weighting coefficients is supplied to the next layers through the intermediate hidden layers. More hidden layers can be added to obtain a quite powerful multilayer network. The MLP architecture has been successfully employed as a universal function approximation in many modelling situations [28].

\section{Generalities on the PV Panel Behaviour}

The electrical power produced by PV devices is linked to the solar irradiance on the panel and the operating temperature, but also depends on the connected electrical load $R_{L}$ as shown in Figure 2; indeed, the load defines the operating point on the $P-V$ characteristic. For given values of irradiance, temperature, and electrical load, the operating point can be identified by drawing on the $P-V$ characteristic the lines of the different $R_{L}$. Therefore, in correspondence with a generic constant load connected to a photovoltaic panel, the working point will move along the load curve under the effect of temperature variations and solar irradiance during the day. The maximum power point (MPP) is identified by a red circle and its coordinates in the $P-V$ plane are $\left(P_{\max }(G, T), V_{\operatorname{mpp}}(G, T)\right)$; in the $I-V$ plane, the coordinates of MPP are $\left(I_{\text {mpp }}(G, T), V_{\text {mpp }}(G, T)\right)$. A careful analysis of $P$ $V$ curves permits to immediately recognize as the electrical behaviour of a generic PV panel can be represented in three modes or regimens:

(i) when the ratio between the working voltage $V$ and the voltage of maximum power $V_{\text {mpp }}$ at given temperature is less than 0.95 , the characteristic $P-V$ is practically 
linear and the power is strongly correlated to the incident solar irradiance; for constant solar irradiance, there is no temperature influence in the power output;

(ii) when the ratio $V / V_{\mathrm{mpp}}$ for a given solar irradiance and temperature is greater than 1.05 , the $P-V$ characteristics of the panel decreases much more rapidly and the influence of solar irradiance becomes less significant (saturation conditions); for constant solar irradiance there is a linear correlation between temperature and the power output;

(iii) the regimen identified by a ratio $0.95<V / V_{\text {mpp }}<$ 1.05 characterizes the state of a PV panel connected to a maximum power point tracking system (MPPT) in which the load dynamically adapts to generate the maximum power (red circle).

\section{Data Acquisition System: Input Data Vector}

To employ and train an ANN, a large database of specific data that represent the analysed physical system is required. To this aim, a test facility was built up on the roof of the Department of Energy, Information Engineering, and Mathematical Models (DEIM) at the University of Palermo. The monitoring system consists of two photovoltaic modules and a pyranometer tilted at $38^{\circ}$ facing south, a precision resistance set used as calibrated load and a multimeter. Concerning the data acquisition of climate parameters, a network of weather stations was built up [33]. The thermal regimen of the PV modules has been measured with thermocouples (type $\mathrm{T}$, copper-constantan) installed at the rear film of the module. All data were collected every 30 minutes and stored for the further calculations and comparisons. The physical data used for the training of the ANN were as follows:

(i) air temperature $T_{\text {air }}\left[{ }^{\circ} \mathrm{C}\right]$;

(ii) cell temperature $T_{c}\left[{ }^{\circ} \mathrm{C}\right]$;

(iii) solar irradiance $G\left[\mathrm{~W} / \mathrm{m}^{2}\right]$;

(iv) wind speed $W[\mathrm{~m} / \mathrm{s}]$;

(v) open circuit voltage $V_{\mathrm{OC}}[\mathrm{V}]$;

(vi) short circuit current $I_{\mathrm{SC}}[\mathrm{A}]$.

These last two parameters are important to improve the evaluation the PV panel power output. Their values are evaluated by using the following expressions [34]:

$$
\begin{gathered}
I_{\mathrm{SC}}=I_{\mathrm{sc}, \mathrm{ref}} \frac{G}{G_{\mathrm{ref}}}+\mu_{I_{\mathrm{SC}}}\left(T_{c}-T_{\mathrm{ref}}\right), \\
V_{\mathrm{OC}}=V_{\mathrm{OC}, \mathrm{ref}}+n T \ln \left(\frac{G}{G_{\mathrm{ref}}}\right)+\mu_{V_{\mathrm{OC}}}\left(T_{c}-T_{\mathrm{ref}}\right),
\end{gathered}
$$

where the subscript ref identifies the reference conditions $\left(G=1000 \mathrm{~W} / \mathrm{m}^{2} ; T=25^{\circ} \mathrm{C}\right)$ and $\mu_{I_{\mathrm{SC}}}$ and $\mu_{V_{\mathrm{OC}}}$ are the short circuit current and open circuit voltage temperature coefficients, respectively [35].

The dataset used for the following analyses consists in more than 6000 data points. The $15 \%$ of data will be used as a test dataset (not used for the ANN training phase).
TABLE 1: Data sheet of Kyocera KC175GH-2.

\begin{tabular}{lcc}
\hline Maximum power & $P_{\max }[\mathrm{W}]$ & 175 \\
Maximum voltage & $V_{\mathrm{mpp}}[\mathrm{V}]$ & 23.6 \\
Maximum current & $I_{\mathrm{mpp}}[\mathrm{A}]$ & 7.42 \\
Open circuit voltage & $V_{\mathrm{OC}}[\mathrm{V}]$ & 29.2 \\
Short circuit current & $I_{\mathrm{SC}}[\mathrm{A}]$ & 8.09 \\
$V_{\mathrm{OC}}$ thermal coefficient & $\mu_{\mathrm{V}_{\mathrm{OC}}}\left[\mathrm{V} /{ }^{\circ} \mathrm{C}\right]$ & -0.109 \\
$I_{\mathrm{SC}}$ thermal coefficient & $\mu_{\mathrm{ISC}_{\mathrm{SC}}}\left[\mathrm{mA} /{ }^{\circ} \mathrm{C}\right]$ & 3.18 \\
\hline
\end{tabular}

TABle 2: Data sheet of Sanyo HIT240HDE4.

\begin{tabular}{lcc}
\hline Maximum power & $P_{\max }[\mathrm{W}]$ & 240 \\
Maximum voltage & $V_{\mathrm{mpp}}[\mathrm{V}]$ & 35.5 \\
Maximum current & $I_{\mathrm{mpp}}[\mathrm{A}]$ & 6.77 \\
Open circuit voltage & $V_{\mathrm{OC}}[\mathrm{V}]$ & 43.6 \\
Short circuit current & $I_{\mathrm{SC}}[\mathrm{A}]$ & 7.37 \\
$V_{\mathrm{OC}}$ thermal coefficient & $\mu_{\mathrm{V}_{\mathrm{OC}}}\left[\mathrm{V} /{ }^{\circ} \mathrm{C}\right]$ & -0.109 \\
$I_{\mathrm{SC}}$ thermal coefficient & $\mu_{\mathrm{ISC}_{\mathrm{SC}}}\left[\mathrm{mA} /{ }^{\circ} \mathrm{C}\right]$ & 2.21 \\
\hline
\end{tabular}

The monitoring campaign involved the measurement of the performances of two different photovoltaic panels: a Kyocera KC175-GH-2 polycrystalline panel and a Sanyo HIT240 HDE4 monocrystalline panel. The principal characteristic of the two panels are showed in Tables 1 and 2.

The measurement campaign about the power output of the PV modules took several months and was characterized by a frequent change of the resistive loads to the aim of acquiring data relating to the entire $P-V$ curve. All data are subject to a preprocessing step that consists in a preliminary analysis that permits to identify possible outliers, to remove uncorrected values, to carry out a statistical analysis, and to perform a correlation analysis.

To simulate the presence of a MPPT device, individual records characterized by a $0.95<V / V_{\text {mpp }}<1.05$ were extracted from the original database.

After the preprocessing step, the database was validated and the correlation analysis has permitted a first evaluation of the mutual relationships among the considered variables.

Figures 6 and 7 show the linear correlation between the power output $P$ and all the other features. The higher the bar goes, the more the features are correlated. In both cases the preliminary correlation analysis identified a strong correlation between $P$ and the solar irradiance; a moderate correlation with air temperature $T_{\text {air }}$ and wind speed was found.

A statistical analysis permitted to assess the maximum (Max), mean (Mean) and minimum (Min) values and the standard deviation (StDev) of all considered features (Tables 3 and 4).

In our study, for the topology of the tested ANN, we decided to use an input vector with six components: $T_{\text {air }}$, $G, T_{\text {cell }}, W, V_{\text {oc }}\left(G, T_{\text {cell }}\right)$, and $I_{\text {sc }}\left(G, T_{\text {cell }}\right)$; the output vector has only one component: the power output $P$, as shown in Figure 8 . 
TABle 3: Preliminary statistics evaluation of weather, thermal, and electric data pertaining Kyocera panel.

\begin{tabular}{|c|c|c|c|c|c|c|}
\hline & $T_{\text {air }}\left[{ }^{\circ} \mathrm{C}\right]$ & $T_{\text {cell }}\left[{ }^{\circ} \mathrm{C}\right]$ & $G\left[\mathrm{~W} / \mathrm{m}^{2}\right]$ & $W[\mathrm{~m} / \mathrm{s}]$ & $I_{\mathrm{SC}}[\mathrm{A}]$ & $V_{\mathrm{OC}}[\mathrm{V}]$ \\
\hline Max & 27.2 & 51.1 & 1078.2 & 7.2 & 8.7 & 30.2 \\
\hline Min & 9.9 & 15.7 & 126.4 & 0 & 1.0 & 26.5 \\
\hline Mean & 19.5 & 36.0 & 729.3 & 2.31 & 5.9 & 28.1 \\
\hline StDev & 2.3 & 7.3 & 293.2 & 1.23 & 2.3 & 0.7 \\
\hline
\end{tabular}

TABLE 4: Preliminary statistics evaluation of weather, thermal, and electric data pertaining Sanyo panel.

\begin{tabular}{lcccccc}
\hline & $T_{\text {air }}\left[{ }^{\circ} \mathrm{C}\right]$ & $T_{\text {cell }}\left[{ }^{\circ} \mathrm{C}\right]$ & $G\left[\mathrm{~W} / \mathrm{m}^{2}\right]$ & $W[\mathrm{~m} / \mathrm{s}]$ & $I_{\mathrm{SC}}[\mathrm{A}]$ & 3.8 \\
\hline Max & 30.9 & 51.8 & 1044.3 & 5.23 & 0.4 & $V_{\mathrm{OC}}[\mathrm{V}]$ \\
Min & 17.8 & 22.9 & 129.8 & 0 & 6.4 \\
Mean & 25.8 & 42 & 725.4 & 2.5 & 2.7 & 63.7 \\
StDev & 1.8 & 6.0 & 259.6 & 1.1 & 0.9 & 0.4 \\
\hline
\end{tabular}

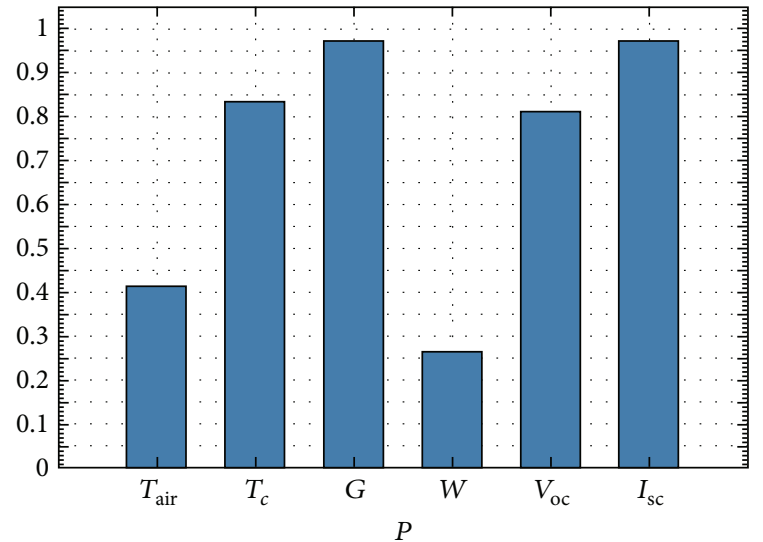

FIGURE 6: Correlation analysis between the power output and all input data of the Kyocera panel.

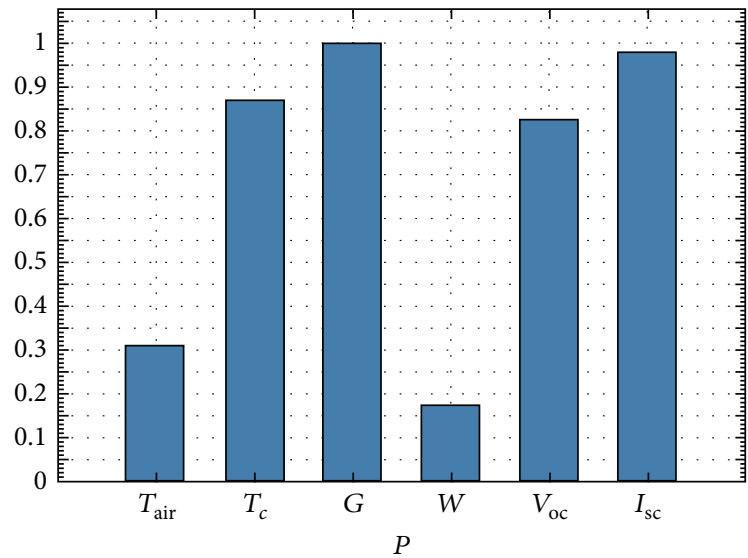

FIGURE 7: Correlation analysis between the power output and all input data of the Sanyo panel.

\section{ANN Topologies}

After the preprocessing phase, the authors explored different topologies of ANN. In the following part, will be described only the best ANN solutions:

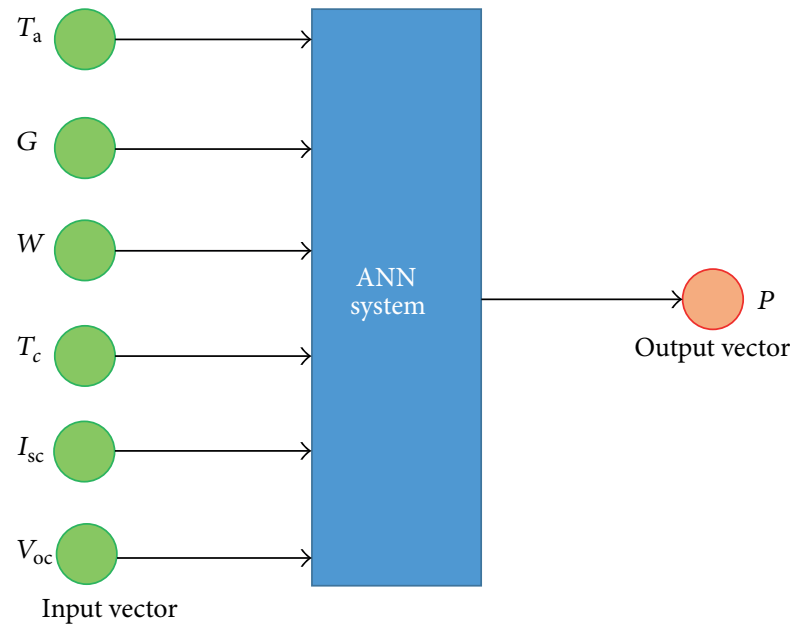

FIGURE 8: Definition of input and output vectors of the tested ANNs.

(i) one hidden layer MLP;

(ii) RNN MLP;

(iii) gamma memory ANN.

For each topology are analysed the design and the algorithm, each neural network was trained and was validated with a post processing phase.

\section{Description of the Implemented ANN Topology}

11.1. One Hidden Layer MLP. The one hidden layer MLP is a kind of ANN consisting of three layers of ANs in a directed graph, with each layer fully connected to the next one. In this work, except for the input ANs, each node is a neuron with a sigmoid activation function and a common supervised learning technique for training the network was used. The tested topology is one of the simplest available for ANNs and is composed by two input sources, two function blocks, two weight layers, one hidden weight layer, and one error criterion block. 


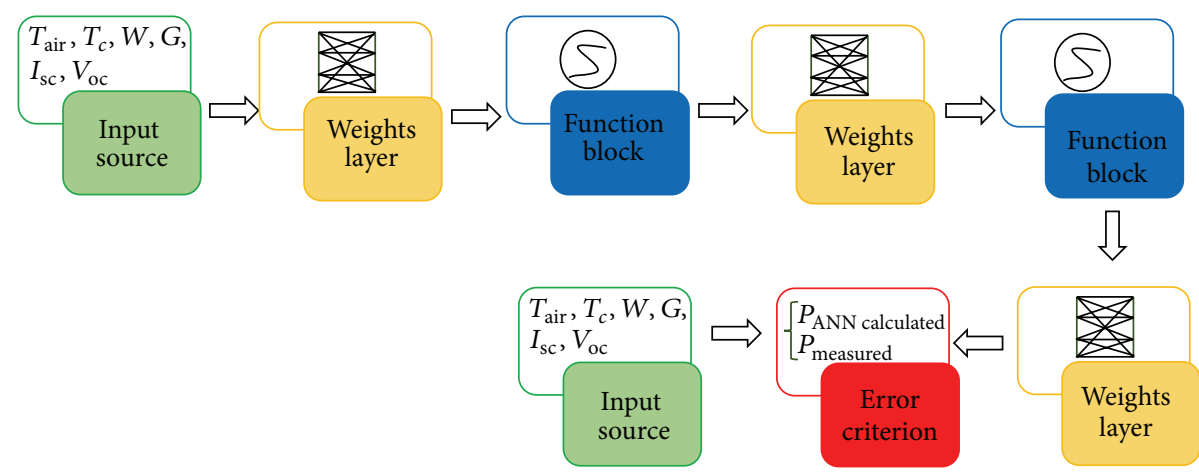

FIGURE 9: Schema of one hidden layer MLP topology for the power output evaluation.

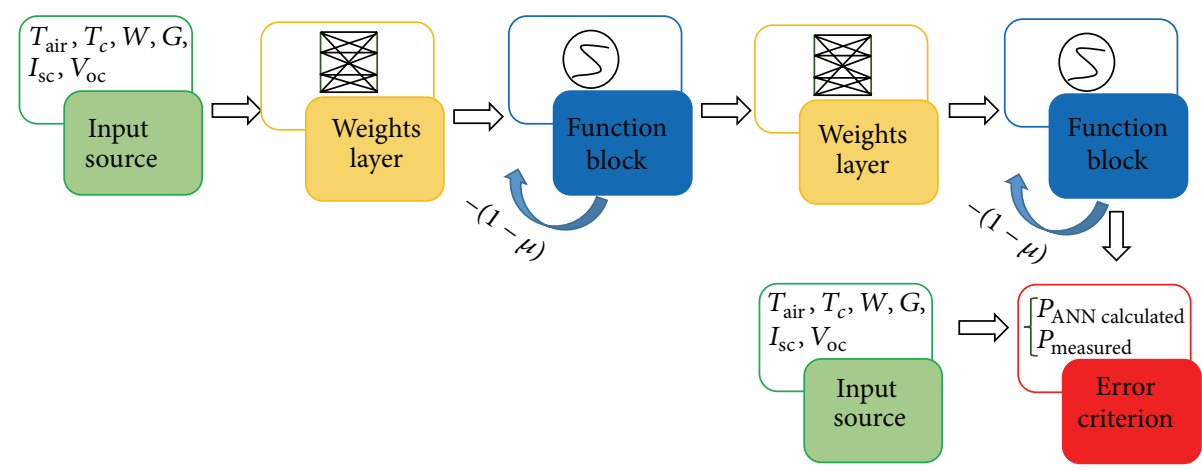

FIgURE 10: Schema of RNN MLP topology for the power output evaluation.

Figure 9 schematizes the tested one hidden layer MLP topology to evaluate power output of a PV panel.

11.2. RNN MLP. The RNN MLP is a simple ANN topology that employs a recursive flow of the signal to preserve and to use the temporal sequence of events as a useful information. This topology is composed of two input sources, two weight layer, one hidden weight layer, two recursive function blocks, and one error criterion.

Figure 10 shows the RNN MLP topology for the power output evaluation. The recursivity is iconized by a feedback connection where $\mu$ is the weight of the feedback used to scale the input. In our test, each signal flowing into the recursive function block is linked to a different value of $\mu$.

11.3. Gamma Memory ANN. The gamma memory (Figure 11) processing element (PE) is used in dynamic systems to remember past signals [36]. It enables the usage of past information to predict current and future states. The gamma neuron is ideal for neural networks since the time axis is scaled by the parameter $\mu$, which can be treated as any weight and adapted using back propagation.

The application of gamma memory permitted to employ an ANN to emulate the $P$ trends. In this work was proposed an ANN constituted by two input sources, three gamma memory blocks, three weight layer, three function blocks, and one error criterion block (Figure 12).

\section{Postprocessing Phase: Performance Assessment of ANNs}

After the training, for each ANN, the postprocessing phase evaluate the difference between the calculated and the measured output vector. The data used for this phase are not used for the training process. The performance assessment is carried out by means of three indexes:

(i) the mean error $(\mathrm{ME})$ is

$$
\mathrm{ME}=\frac{1}{N} \sum_{i=1}^{N}\left(P_{\text {measured }, i}-P_{\mathrm{ANN} \text { calculated }, i}\right)
$$

where $N$ is the number of samples,

(ii) the mean absolute error (MAE) represents the quantity used to measure how close forecasts or predictions are to the eventual outcome:

$$
\text { MAE }=\frac{1}{N} \sum_{i=1}^{N}\left|P_{\text {measured }, i}-P_{\mathrm{ANN} \text { calculated }, i}\right| ;
$$

(iii) the standard deviation $\sigma$ shows how much variation or "dispersion" exists from the average (mean or expected value). A low standard deviation indicates that the sample data tend to be very close to the mean; 


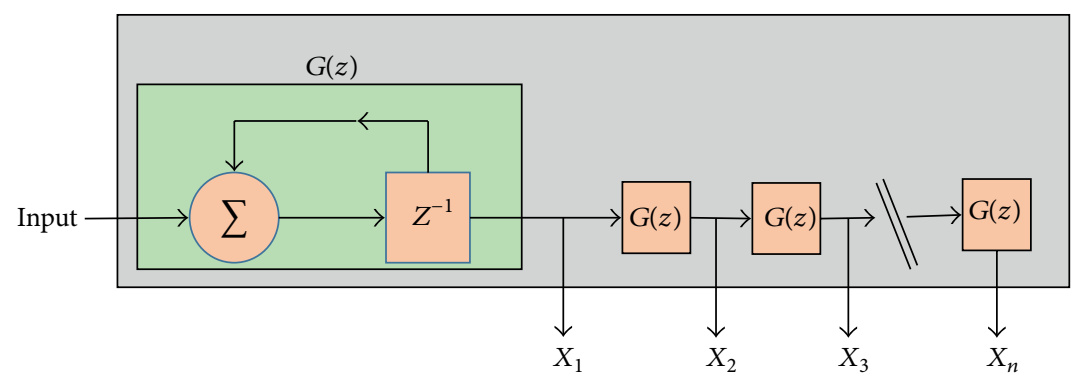

FIGURE 11: Schema of the gamma memory processing element topology.

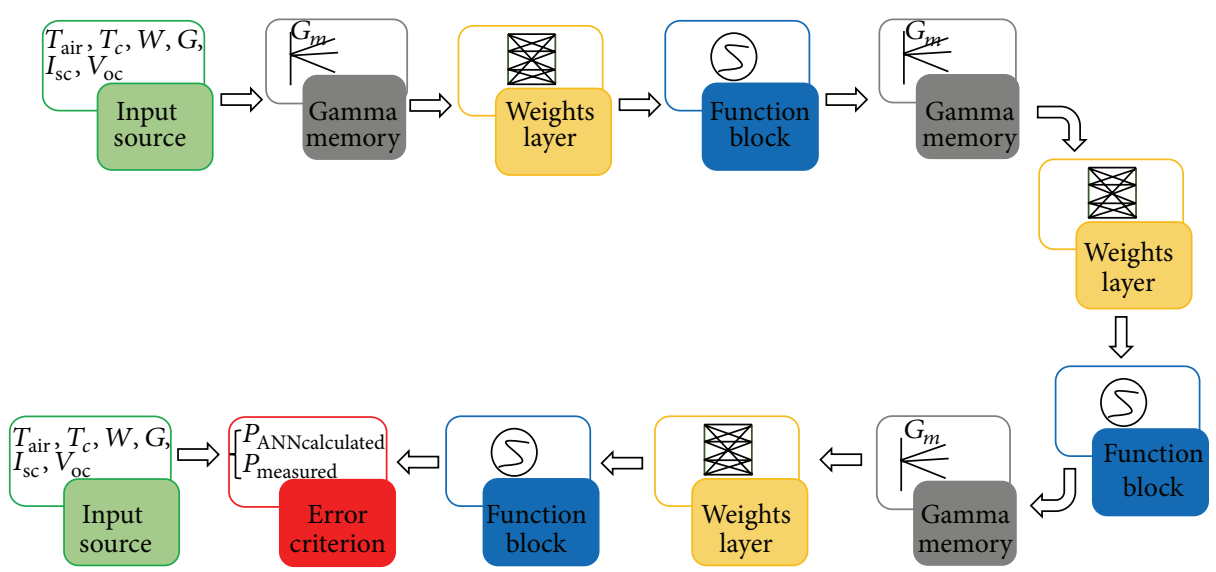

FIGURE 12: Schema of gamma memory topology for the power output evaluation.

high standard deviation indicates that data are spread out over a large range of values:

$$
\sigma=\sqrt{\frac{1}{N-1} \sum_{i=1}^{N}\left(P_{\text {measured }, i}-\bar{P}_{\text {ANN calculated }, i}\right)^{2}} .
$$

\section{Results and Discussions}

As previously described, each ANN was characterized by a training phase, a postprocessing phase evaluates the error, and the absolute error between the measured and the calculated operating temperature data. To better analyse the validity of the ANN, different simulations were carried out changing the time of the training phase and/or the epochs. In all cases, the training phase has been suspended in order to avoid the over-fitting. Furthermore, for each topology was identified the confidence plot that contains the $95 \%$ of the outputs.

To better understand how ANNs performance can be evaluated, Figure 13 shows the calculated power output versus measured power output (data points not used for training phase).

In Tables 5 and 6, the results of several ANNs tested topologies are reported.

The result coming from the ANNs designed to predict the power output produced by a PV panel shows that this kind of approach is very promising. Mean errors appear to be generally very low $(1 \mathrm{~W})$. ANN topologies based on MLP

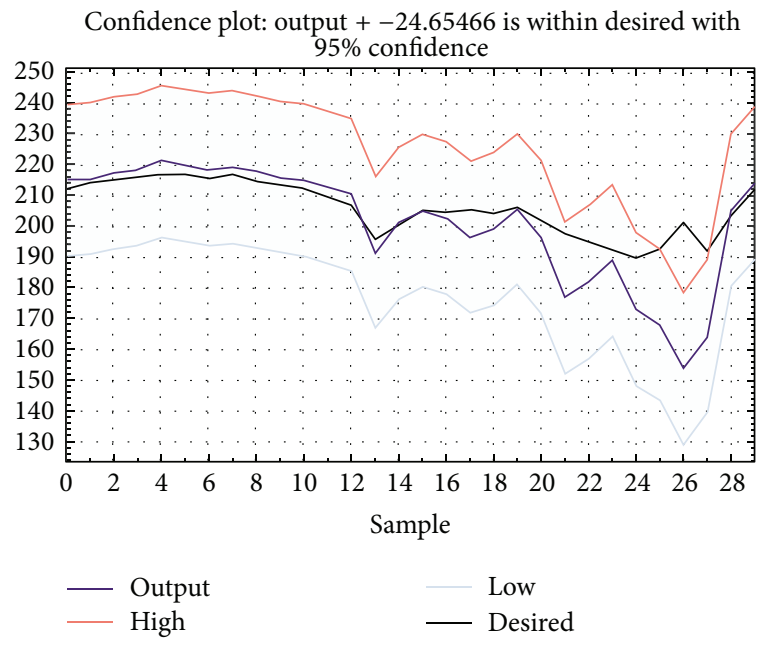

FIGURE 13: Calculated power output versus measured power output for the Sanyo module (MlP_1 topology).

for both panels were very good in terms of prediction error even if they required a longer time for the training phase. The results of the RNNs and gamma memory ANNs are characterized by good performances with shorter training time for the Kyocera module. The Sanyo panel has generally required longer training time but with excellent results in term of mean error especially with the gamma memory ANN. 
TABLE 5: ANNs results for the Kyocera panel; bold identifies the ANNs with the best performance.

\begin{tabular}{|c|c|c|c|c|c|c|c|c|}
\hline \multirow{2}{*}{ Topology } & \multicolumn{3}{|c|}{ Error distribution [W] } & \multicolumn{3}{|c|}{ Absolute error distribution [W] } & \multirow{2}{*}{ Epochs } & \multirow{2}{*}{ Time $[\mathrm{s}]$} \\
\hline & Mean & Median & Stdev & Mean & Median & Stdev & & \\
\hline Mlp_1 & 0.05 & -0.5 & 8.1 & 5.3 & 3.0 & 6.2 & 15417 & 31 \\
\hline Mlp_2 & -0.1 & 0.5 & 7.3 & 4.3 & 2.3 & 5.9 & 2854 & 5 \\
\hline Mlp_3 & -1.9 & -1.1 & 8.1 & 5.3 & 3.0 & 6.4 & 6354 & 12 \\
\hline Mlp_4 & -0.9 & -0.3 & 7.6 & 4.6 & 2.8 & 6.1 & 993 & 1 \\
\hline RNN_1 & -0.6 & -0.6 & 4.8 & 3.3 & 2.1 & 3.6 & 4976 & 102 \\
\hline RNN_2 & 9.8 & 7.1 & 11.2 & 11.2 & 8.2 & 9.8 & 533 & 10 \\
\hline RNN_3 & 0.7 & 1.4 & 8.6 & 5.7 & 3.2 & 6.5 & 555 & 11 \\
\hline Gamma_1 & -1.0 & 0.4 & 8.9 & 5.8 & 3.2 & 6.8 & 126 & 2 \\
\hline Gamma_2 & -3.0 & -1.5 & 8.3 & 5.7 & 3.4 & 6.7 & 346 & 6 \\
\hline
\end{tabular}

TABLE 6: ANNs results for the Sanyo panel; bold identifies the ANNs with the best performance.

\begin{tabular}{|c|c|c|c|c|c|c|c|c|}
\hline \multirow{2}{*}{ Topology } & \multicolumn{3}{|c|}{ Error distribution [W] } & \multicolumn{3}{|c|}{ Absolute error distribution [W] } & \multirow{2}{*}{ Epochs } & \multirow{2}{*}{ Time $[s]$} \\
\hline & Mean & Median & Stdev & Mean & Median & Stdev & & \\
\hline Mlp_1 & -0.1 & -0.8 & 9.1 & 4.9 & 3.0 & 7.8 & 3162 & 3 \\
\hline Mlp_2 & -3.8 & -3.1 & 5.3 & 4.6 & 3.4 & 4.7 & 16176 & 16 \\
\hline RNN_1 & -1.3 & -0.1 & 10.1 & 5.7 & 3.8 & 8.4 & 3361 & 29 \\
\hline RNN_2 & -1.7 & 0.04 & 10.3 & 5.9 & 4.0 & 8.6 & 305 & 3 \\
\hline Gamma_1 & 0.02 & 0.4 & 9.4 & 6.01 & 4.5 & 7.3 & 182 & 9 \\
\hline Gamma_2 & 0.2 & 0.7 & 5.9 & 4.5 & 4.0 & 3.8 & 3134 & 27 \\
\hline
\end{tabular}

\section{Conclusions}

In the paper, different network architectures have been tested in order to forecast the electric power generated by a PV module in real conditions. Data used to train the networks were acquired using two different types of PV modules connected to calibrated electrical loads. Climatic variables were acquired by means of a weather station. The performances evaluation of the ANNs was performed by comparing the prediction with the real power output and the errors were generally contained within the $0.05-1 \%$ of the module peak power output. ANNs with simpler architecture generally required longer training time while more complex ANNs have requested shorter training time. Results show that adaptive techniques are able to predict the power output of a PV panel with great accuracy and short computational time. These algorithms can play a dominant role concerning remote management of PV in a probable future when this technology will be extremely widespread in the territory.

\section{Nomenclature}

$A_{i}: \quad$ Activation potential

AN: Artificial neuron

ANN: Artificial neural network

$b_{i}$ : Bias coefficient

FLCs: Fuzzy logic controllers

$G: \quad$ Solar irradiance $\left[\mathrm{W} / \mathrm{m}^{2}\right]$

I: $\quad$ Current [A]

$I_{0}$ : Diode reverse saturation current [A]
$I_{\mathrm{mpp}}: \quad$ Maximum current $[\mathrm{A}]$

$I_{L}: \quad$ Photocurrent [A]

$I_{\text {sc }}: \quad$ Short circuit current [A]

$k: \quad$ Scale parameter

$k_{i}$ : $\quad$ Constants of current proportionality

$k_{v}$ : Constants of voltage proportionality

MPP: Maximum Power Point

MPPT: Maximum Power Point technique

$n: \quad$ Ideality factor

$N$ : Number of elements in the input vector

$P$ : $\quad$ Power output [W]

PV: Photovoltaic

$R_{L}: \quad$ Electric load $[\Omega]$

RNN: Radial neural network

$R_{\mathrm{sh}}: \quad$ Shunt resistance $[\Omega]$

$R_{s}: \quad$ Series resistance $[\Omega]$

$T_{\text {air }}: \quad$ Air temperature $\left[{ }^{\circ} \mathrm{C}\right]$

$T_{c}$ : Cell absolute temperature $\left[{ }^{\circ} \mathrm{C}\right]$

$V: \quad$ Voltage [V]

$V_{\mathrm{mpp}}: \quad$ Maximum voltage [V]

$V_{\text {oc }}: \quad$ Open circuit voltage $[\mathrm{V}]$

$\omega_{i j}: \quad$ Weights

$W: \quad$ Wind speed $[\mathrm{m} / \mathrm{s}]$

$x_{i}: \quad$ Interconnection

$y_{i}: \quad$ Neuron output

$\mu_{I_{\mathrm{SC}}}: \quad$ Short circuit current temperature coefficients $\left[\mathrm{mA} /{ }^{\circ} \mathrm{C}\right]$

$\mu_{V_{\mathrm{OC}}}: \quad$ Open circuit voltage temperature coefficients $\left[\mathrm{V} /{ }^{\circ} \mathrm{C}\right]$. 


\section{Conflict of Interests}

The authors declare that there is no conflict of interests regarding the publication of this paper.

\section{References}

[1] V. Vossos, K. Garbesi, and H. Shen, "Energy savings from directDC in US residential buildings," Energy and Buildings, vol. 68, pp. 223-231, 2014.

[2] W. D. Thomas and J. J. Duffy, "Energy performance of net-zero and near net-zero energy homes in New England," Energy and Buildings, vol. 67, pp. 551-558, 2013.

[3] M. Cellura, L. Campanella, G. Ciulla et al., "The redesign of an Italian building to reach net zero energy performances: a case study of the SHC Task 40-ECBCS Annex 52," in Proceedings of the ASHRAE Transactions, vol. 117, part 2, pp. 331-339, June 2011.

[4] J. G. Kang, J. H. Kim, and J. T. Kim, "Performance evaluation of DSC windows for buildings," International Journal of Photoenergy, vol. 2013, Article ID 472086, 6 pages, 2013.

[5] F. Asdrubali, F. Cotana, and A. Messineo, "On the evaluation of solar greenhouse efficiency in building simulation during the heating period," Energies, vol. 5, no. 6, pp. 1864-1880, 2012.

[6] C. Rodriguez and G. A. J. Amaratunga, "Dynamic stability of grid-connected photovoltaic systems," in Proceedings of the IEEE Power Engineering Society General Meeting, pp. 2193-2199, June 2004.

[7] L. Wang and Y.-H. Lin, "Random fluctuations on dynamic stability of a grid-connected photovoltaic array," in Proceedings of the IEEE Power Engineering Society Winter Meeting, vol. 3, pp. 985-989, February 2001.

[8] Y. T. Tan and D. S. Kirschen, "Impact on the power system of a large penetration of photovoltaic generation," in Proceedings of the IEEE Power Engineering Society General Meeting, pp. 1-8, June 2007.

[9] E. Skoplaki and J. A. Palyvos, "On the temperature dependence of photovoltaic module electrical performance: a review of efficiency/power correlations," Solar Energy, vol. 83, no. 5, pp. 614-624, 2009.

[10] V. Salas, E. Olías, A. Barrado, and A. Lázaro, "Review of the maximum power point tracking algorithms for stand-alone photovoltaic systems," Solar Energy Materials and Solar Cells, vol. 90, no. 11, pp. 1555-1578, 2006.

[11] T. Esram and P. L. Chapman, "Comparison of photovoltaic array maximum power point tracking techniques," IEEE Transactions on Energy Conversion, vol. 22, no. 2, pp. 439-449, 2007.

[12] J. Surya Kumari and C. Sai Babu, "Comparison of maximum power point tracking algorithms for photovoltaic system," International Journal of Advances in Engineering and Technology, vol. 1, no. 5, pp. 133-148, 1963.

[13] M. A. S. Masoum, H. Dehbonei, and E. F. Fuchs, "Theoretical and experimental analyses of photovoltaic systems with voltage- and current-based maximum power-point tracking," IEEE Transactions on Energy Conversion, vol. 17, no. 4, pp. 514$522,2002$.

[14] J. Ahmad and H.-J. Kim, "A voltage based maximum power point tracker for low power and low cost photovoltaic applications," World Academy of Science, Engineering and Technology, vol. 60, pp. 714-717, 2009.

[15] V. Lo Brano and G. Ciulla, "An efficient analytical approach for obtaining a five parameters model of photovoltaic modules using only reference data," Applied Energy, vol. 111, pp. 894-903, 2013.

[16] M. Veerachary, T. Senjyu, and K. Uezato, "Neural-networkbased maximum-power-point tracking of coupled-inductor interleaved-boost-converter-supplied PV system using fuzzy controller," IEEE Transactions on Industrial Electronics, vol. 50, no. 4, pp. 749-758, 2003.

[17] B. M. Wilamowski and J. Binfet, "Microprocessor implementation of fuzzy systems and neural networks," in Proceedings of the International Joint Conference on Neural Networks (IJCNN '01), vol. 1, pp. 234-239, Washington, DC, USA, July 2001.

[18] C.-Y. Won, D.-H. Kim, S.-C. Kim, W.-S. Kim, and H.-S. Kim, "New maximum power point tracker of photovoltaic arrays using fuzzy controller," in Proceedings of th 25th Annual IEEE Power Electronics Specialists Conference (PESC '94), vol. 1, pp. 396-403, June 1994.

[19] A. E.-S. A. Nafeh, F. H. Fahmy, and E. M. Abou El-Zahab, "Evaluation of a proper controller performance for maximumpower point tracking of a stand-alone PV system," Solar Energy Materials and Solar Cells, vol. 75, no. 3-4, pp. 723-728, 2003.

[20] N. Patcharaprakiti, S. Premrudeepreechacharn, and Y. Sriuthaisiriwong, "Maximum power point tracking using adaptive fuzzy logic control for grid-connected photovoltaic system," Renewable Energy, vol. 30, no. 11, pp. 1771-1788, 2005.

[21] T. Hiyama, S. Kouzuma, and T. Imakubo, "Identification of optimal operating point of PV modules using neural network for real time maximum power tracking control," IEEE Transactions on Energy Conversion, vol. 10, no. 2, pp. 360-367, 1995.

[22] T. Hiyama, S. Kouzuma, T. Imakubo, and T. H. Ortmeyer, "Evaluation of neural network based real time maximum power tracking controller for PV system," IEEE Transactions on Energy Conversion, vol. 10, no. 3, pp. 543-548, 1995.

[23] T. Hiyama and K. Kitabayashi, "Neural network based estimation of maximum power generation from PV module using environmental information," IEEE Transactions on Energy Conversion, vol. 12, no. 3, pp. 241-246, 1997.

[24] A. Cocconi and W. Rippel, "Lectures from GM sunracer case history, lecture 3-1: the Sunracer power systems," Number M101, Society of Automotive Engineers, Warderendale, Pa, USA, 1990.

[25] G. Ciulla, V. Lo Brano, and E. Moreci, "Forecasting the cell temperature of PV modules with an adaptive system," International Journal of Photoenergy, vol. 2013, Article ID 192854, 10 pages, 2013.

[26] V. Lo Brano, G. Ciulla, and M. Beccali, "Application of adaptive models for the determination of the thermal behaviour of a photovoltaic panel," in Proceedings of the International Conferences on Computational Science and Its Applications (ICCSA '13), pp. 344-358, Springer, Ho Chi Minh City, Vietnam, 2013.

[27] K. S. Yigit and H. M. Ertunc, "Prediction of the air temperature and humidity at the outlet of a cooling coil using neural networks," International Communications in Heat and Mass Transfer, vol. 33, no. 7, pp. 898-907, 2006.

[28] M. T. Hagan, H. B. Demuth, and M. Beale, Neural Network Design, PWS Publishing Company, Boston, Mass, USA, 1995.

[29] S. Danaher, S. Datta, I. Waddle, and P. Hackney, "Erosion modelling using Bayesian regulated artificial neural networks," Wear, vol. 256, no. 9-10, pp. 879-888, 2004.

[30] S. Haykin, Neural Networks: A Comprehensive Foundation, MacMillan, New York, NY, USA, 1994. 
[31] V. Pacelli and M. Azzollini, "An artificial neural network approach for credit risk management," Journal of Intelligent Learning Systems and Applications, vol. 3, no. 2, pp. 103-112, 2011.

[32] E. Angelini, G. di Tollo, and A. Roli, "A neural network approach for credit risk evaluation," Quarterly Review of Economics and Finance, vol. 48, no. 4, pp. 733-755, 2008.

[33] V. Lo Brano, A. Orioli, G. Ciulla, and S. Culotta, "Quality of wind speed fitting distributions for the urban area of Palermo, Italy," Renewable Energy, vol. 36, no. 3, pp. 1026-1039, 2011.

[34] V. Lo Brano, A. Orioli, and G. Ciulla, "On the experimental validation of an improved five-parameter model for silicon photovoltaic modules," Solar Energy Materials and Solar Cells, vol. 105, pp. 27-39, 2012.

[35] V. Lo Brano, A. Orioli, G. Ciulla, and A. di Gangi, "An improved five-parameter model for photovoltaic modules," Solar Energy Materials and Solar Cells, vol. 94, no. 8, pp. 1358-1370, 2010.

[36] J. C. Principe, N. R. Euliano, and W. C. Lefebvre, Neural and Adaptive Systems: Fundamentals Through Simulations, John Wiley \& Sons, New York, NY, USA, 1999. 

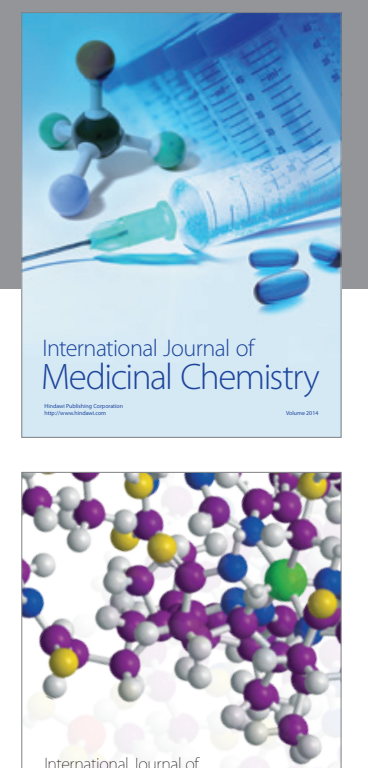

\section{Carbohydrate} Chemistry

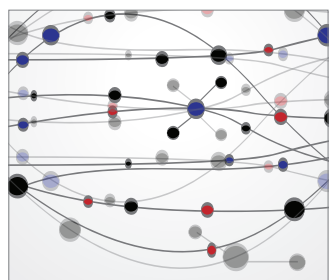

The Scientific World Journal
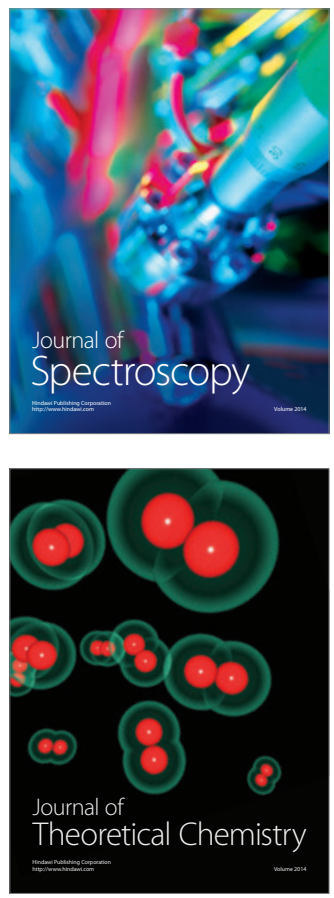
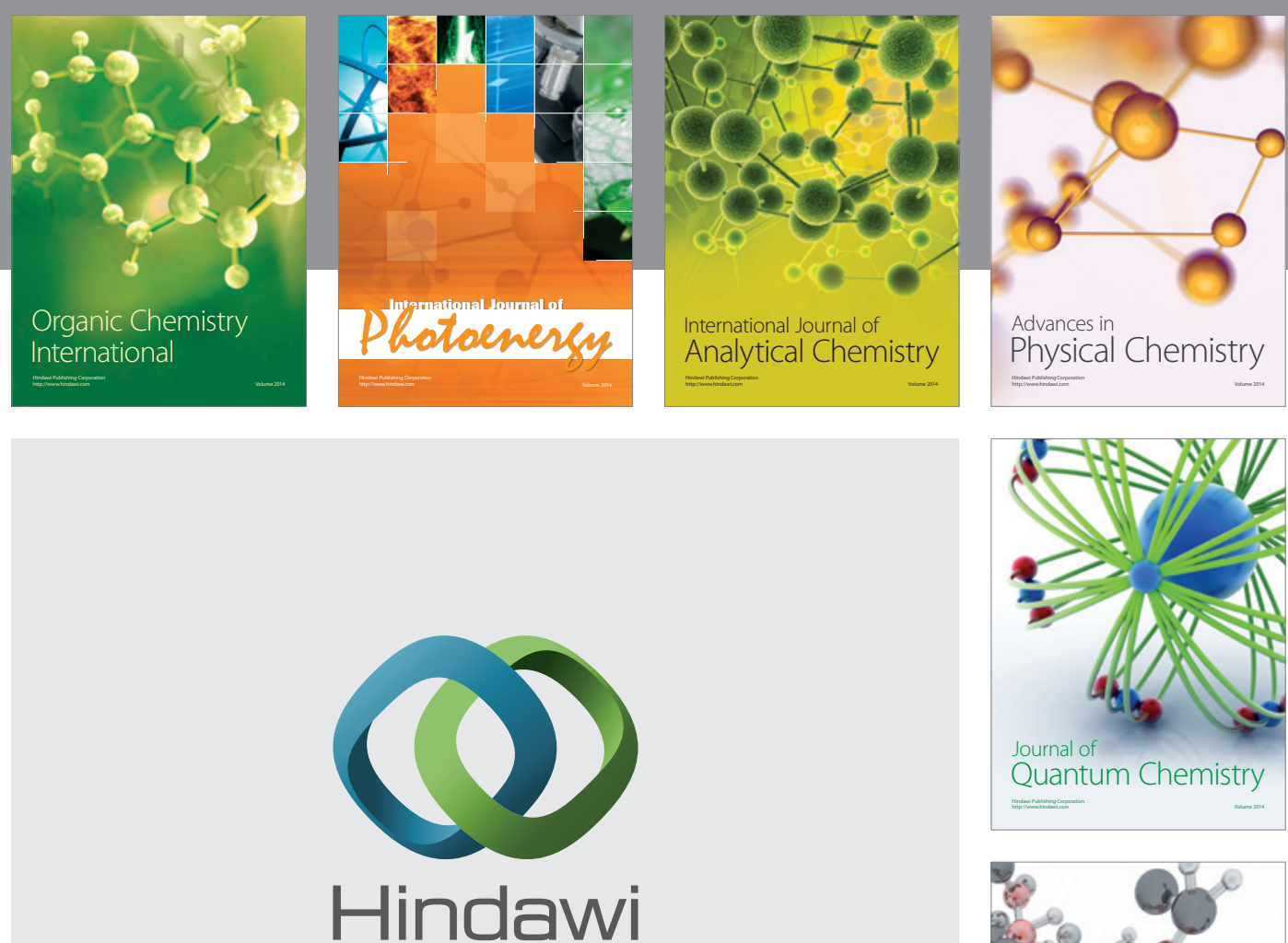

Submit your manuscripts at

http://www.hindawi.com

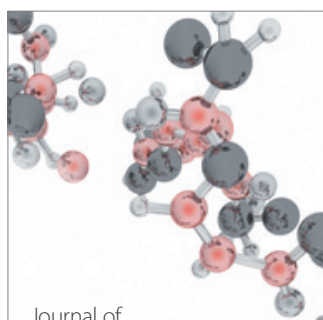

Analytical Methods

in Chemistry

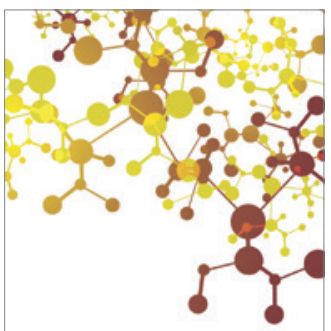

Journal of

Applied Chemistry

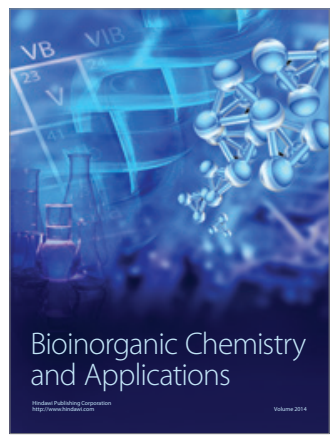

Inorganic Chemistry
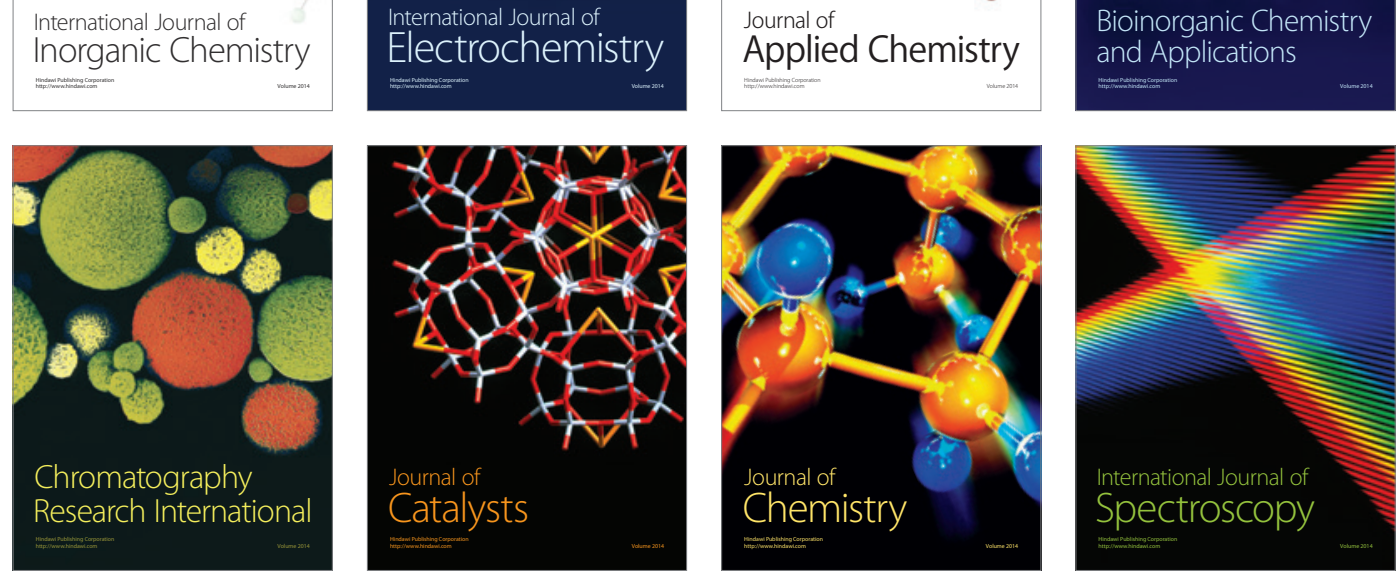